\title{
Evaluating transport time in emergency medical service via GIS: an observational study of Tokyo
}

Tianqi Xia ${ }^{\mathrm{a}, \mathrm{b}}$, Shuzhe Huang ${ }^{\mathrm{b}}$, Xuan Song ${ }^{\mathrm{b}, *}$, Ruochen $\mathrm{Si}^{\mathrm{b}}$, Xiaoya Song ${ }^{\mathrm{b}, \mathrm{c}}$, Ryosuke Shibasaki ${ }^{\mathrm{b}}$, Kyoung-Sook Kim ${ }^{\text {a }}$

\author{
${ }^{a}$ Artificial Intelligence Research Center, National Institute of Advanced Industrial Science and Technology, Tianqi Xia, \\ xiatianqi@csis.u-tokyo.ac.jp,Xuan Song,songxuan@csis.u-tokyo.ac.jp \\ ${ }^{b}$ Centerfor Spatial Information Science, The University of Tokyo, Shuzhe Huang,hgsuze@csis.u-tokyo.ac.jp, Ruochen Si, si@csis.u- \\ tokyo.ac.jp, Ryosuke Shibasaki,shiba@csis.u-tokyo.ac.jp \\ c Heilongjiang Cold Region Urban-Rural Human Settlements Science Key Laboratory, School of Architecture, Harbin Institute of \\ Technology, Xiaoya Song,xy.song8989@gmail.com \\ * Corresponding author
}

Keywords: Spatial Analysis, Emergency Medical Service, Geostatisics

\begin{abstract}
:
Emergency medical service (EMS) is one kind of medical services which focuses on providing first-time rescue to victims of sudden and life-threatening emergencies. Since a lot of studies have pointed out a close relation between the increase of cost time before the patient accommodated to the hospital and the increased risk of mortality, a reasonable distribution of EMS facilities can shorten the transportation time from the scene to the hospital and is critical to ensure the quality of the EMS system.
\end{abstract}

With the development of geographic information science and technology, GIS provides the visualization and analysis approaches for the distribution of the EMS cases, ambulance cars and hospitals as well as the ability of measuring road network distance, which results in the popularity of research with GIS based analysis approaches in the field of public health and EMS system. Despite of the prosperity in such kind of studies, most of them focused on evaluating EMS system by observational case analysis while paying less attention on the emergency medical resource distribution.

With the concerns we mentioned above, this research conducts spatial and temporal analysis for evaluating the transportation time via several GIS methods and take the EMS cases in Tokyo 23 wards as a case study. In addition to the observational studies with EMS case data and several spatial and temporal factors, we pay more attention on evaluating the distribution of cases and hospitals from both sides of demand and supplement. In addition, we also check several assumptions that are widely used in accessibility analysis on public health. As far as we know, our work is first research on detailed hospital distribution analysis in Tokyo area based on observations.

The data source we utilized in our research is the EMS data of Tokyo 23 wards in 2017 provided by Tokyo Fire Department with detailed records of transport time and hospital address. The locations of the cases are specific to 931 basic regions in town and street level with which we map the data to the geometrical region data. In 2017, there are 624,062 EMS cases in Tokyo 23 wards while 550,309 cases have the records of sending to hospital and 429,584 cases have the records of detailed hospital address. The distribution of medical cases is shown in Figure 1. To get hospital points, the hospital address in the dataset are geocoded to the coordinates and matched to the hospital data of Japanese Medical Analysis Platform to acquire the scale information such as the bed numbers and the doctor numbers of the hospital. In Tokyo 23 wards, there are 231 hospitals that are qualified for emergency service while only 214 hospitals have the records in our dataset with the location, scale and case number shown in Figure 2.

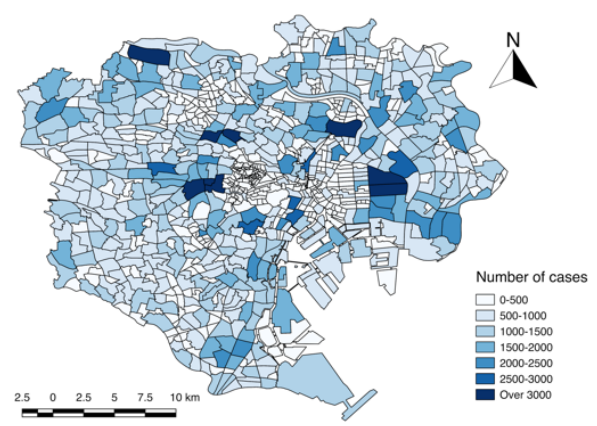

Figure 1. The choropleth map of EMS case number in Tokyo area.

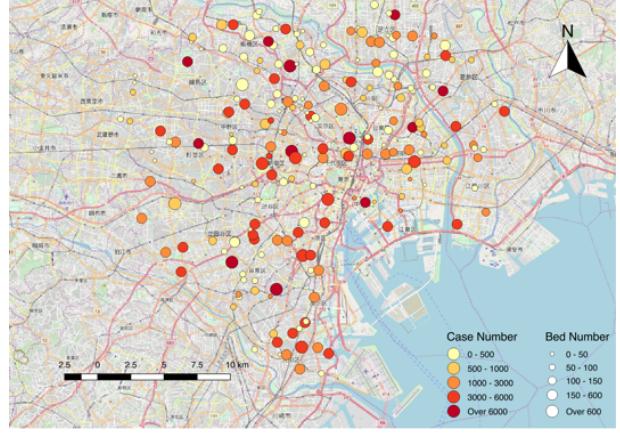

Figure 2. Map of hospital spatial distribution and scale 
From the perspective of spatial information, in EMS case there are three kinds of spatial information with different geometry types. The emergency hospitals can be represented by individual points; thus their distribution can be analyzed through the point patterns. The scene of the case is aggregated to the basic region, thus the travel cost in each region can represent the EMS accessibility of this region. Last but not least, the connection of scene and hospital can be represented by the links in a network space or Euclidean space. Compared to the point and regional data, the links can better reflect both sides of supply and demand, thus is more precise for evaluating the hospitals and regions in EMS system. In this research, we conduct the spatial analysis from two perspectives: the hospital side and the region side. For each side, we firstly conduct spatial analysis on the corresponding data, and then utilize the link data for further analysis.

In this research, Ripley's K-function is utilized to analyze the emergency hospital distribution and the result is shown in Figure 3. The plot indicates that the distribution of hospitals is clustered under almost all distances, which implies that the spatial distribution of hospital is unbalanced. To analyze the spatial inequality into details, we build the Voronoi diagram for the hospitals in Tokyo area. The Voronoi diagram is shown in Figure 6. Notice that since each tessellation represents the range that is nearest to the hospital, the figure also shows the largest distance from the edge of each tessellation to the hospital point. As the map points out, the hospitals in central area is accessible with a rather short distance while in peripheral area, it is probably that the distance to the nearest hospital is very long, which further reveals the imbalance of hospital distribution. In order to analyze the spatial distribution of this imbalance, we calculate the residual error of the regression result on the logarithm of bed and case number with the equation: res $=\log (y+1)-(w \cdot \log (x+1)+b)$ where $y$ is the case number and $x$ is the bed number, $w$ and $b$. From the map we can find an oversupply for the hospitals in central Tokyo area and an overdemand for the hospitals in peripheral area.

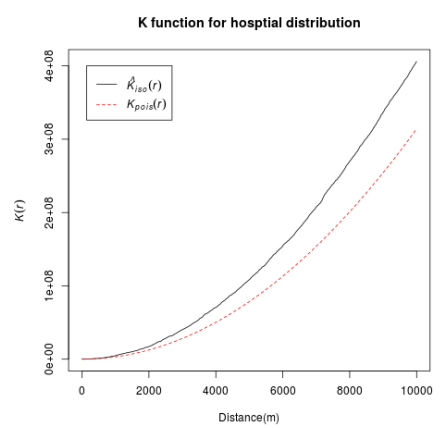

Figure 3. K function result on hospital distribution

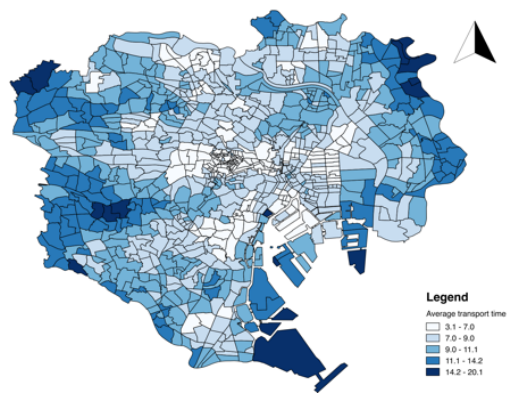

Figure 6. Choropleth map of average transport time

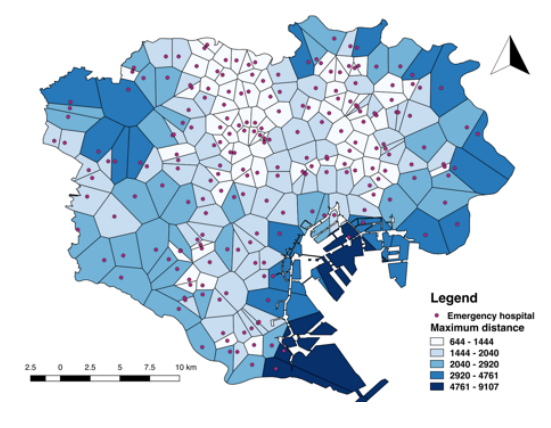

Figure 4. Voronoi diagram of hospitals in Tokyo area

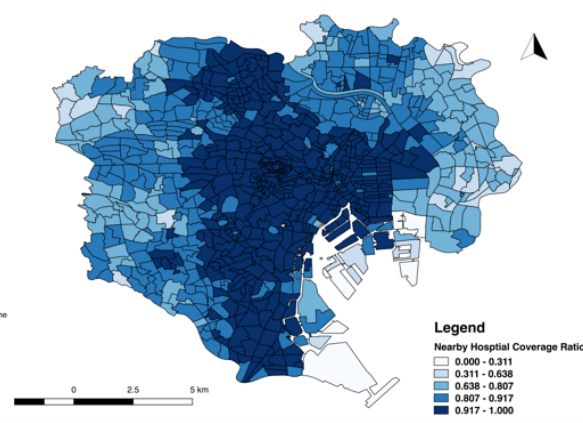

Figure 7. Nearby hospital coverage ratio with 5-kilometer buffers

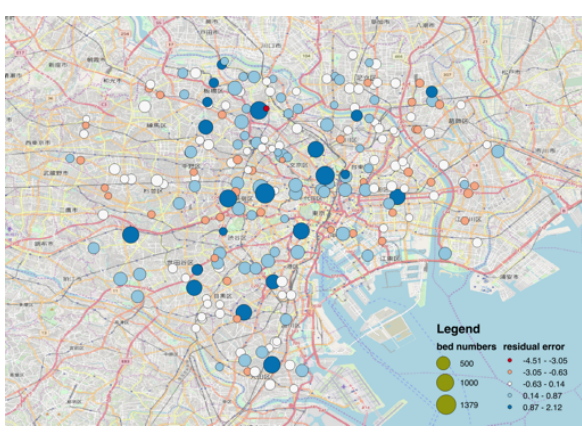

Figure 5. Residual error of regression between case number and bed number

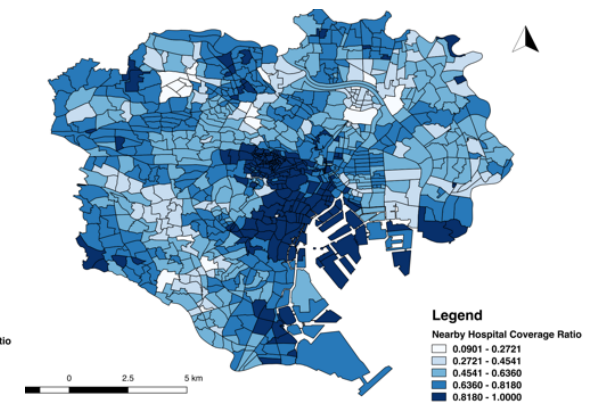

Figure 8. Nearby hospital coverage ratio with nearest five hospitals

From the perspective of regional variance in transport time, a map of average time is shown in Figure 10. Generally, the average cost time in peripheral regions is larger than the central regions and it shares a very similar distribution pattern with the Voronoi diagram of hospital distribution, which implies that in those area the large time cost is probably due to a poor accessibility to the nearby hospitals. To descriptively analyze this feature, we calculate the case ratio for choosing a farther hospital (within 5 kilometers or belongs to nearest 5 hospitals) in each region as shown in Figure. From the two maps we can found that the coverage ratio patterns are different when utilizing different kinds of spatial context. On one hand, when considering the distance ratio with a distancebased neighborhood, we can find that medical resource for the regions in central area can meet the requirement of most cases while in peripheral area even though in some regions they can get access to the nearby hospital quickly, the patients in some cases still need to be sent to a hospital (mainly in central area) with long distance. 